\title{
Ulnar-sided wrist pain. Part I: anatomy and physical examination
}

\author{
Peter S. Vezeridis • Hiroshi Yoshioka • Roger Han • \\ Philip Blazar
}

Received: 12 April 2009/Revised: 26 July 2009 /Accepted: 27 July 2009/Published online: 1 September 2009

(C) The Author(s) 2009. This article is published with open access at Springerlink.com

\begin{abstract}
Ulnar-sided wrist pain is a common complaint, and it presents a diagnostic challenge for hand surgeons and radiologists. The complex anatomy of this region, combined with the small size of structures and subtle imaging findings, compound this problem. A thorough understanding of ulnarsided wrist anatomy and a systematic clinical examination of this region are essential in arriving at an accurate diagnosis. In part I of this review, ulnar-sided wrist anatomy and clinical examination are discussed for a more comprehensive understanding of ulnar-sided wrist pain.
\end{abstract}

Keywords Wrist · Anatomy C Clinical examination ·

Magnetic resonance imaging (MRI)

\section{Introduction}

Pain at the ulnar aspect of the wrist is a diagnostic challenge for hand surgeons and radiologists, due to the small and complex anatomic structures involved. Imaging evidence of the underlying cause of pain is frequently

\section{P. S. Vezeridis $\cdot$ P. Blazar}

Department of Orthopaedic Surgery,

Brigham and Women's Hospital,

Boston, MA, USA

H. Yoshioka $(\bowtie)$

Department of Radiological Sciences,

University of California, Irvine,

100 The City Drive, South Building 56, Suite 300,

Orange, CA 92868, USA

e-mail: hiroshi@uci.edu

\section{R. Han}

Department of Radiology, Brigham and Women's Hospital,

75 Francis Street,

Boston, MA 02115, USA subtle, involving small structures. Familiarity with clinical findings and pertinent anatomy would considerably aid in making accurate diagnoses. In part I of this review, ulnarsided wrist anatomy and clinical examination are reviewed.

\section{Normal anatomy}

Bones and joints

The wrist consists of the distal radius, the ulna, the carpal bones, and the bases of the metacarpals. The construct of two rows of carpal bones that form smooth arcs permits the normal range of wrist motion. Gilula identified three arcs (Gilula's arcs) that circumscribe the proximal (first arc) and distal (second arc) joint surfaces of the first carpal row (scaphoid, lunate and triquetral) and the opposing joint surface formed by the convexity of the capitate and hamate bones (third arc) [1] (Fig. 1).

At the ulnar aspect of the wrist, the distal ulna articulates with the triangular fibrocartilage complex (TFCC). There are two eminences at the distal ulna: the head and the styloid process. Laterally, the head of the ulna is a large round articular eminence with a distally oriented oval articular surface and a horizontal orientation that articulates with the radius. This articulation moves anteriorly and posteriorly due to motion at the distal radioulnar joint during pronation and supination. The styloid process arises from the medial and posterior aspect of the distal ulna, is non-articular in nature, and is the point of origin of the ulnar collateral ligament. The styloid process is separated from the head by a shallow depression where the triangular fibrocartilage attaches.

Ulnar variance refers to the lengths of the distal articular surfaces of the radius and ulna [2]. This variance affects the 


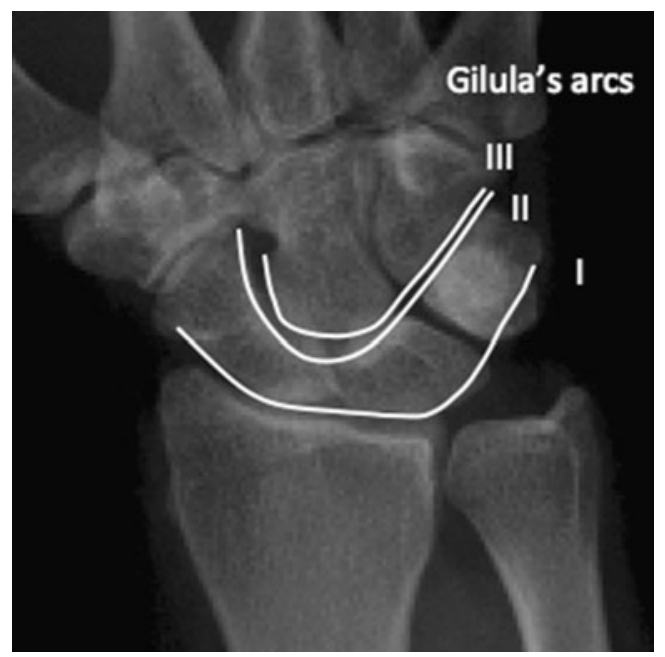

Fig. 1 Radiograph showing Gilula's arcs. Three smooth arcs normally outline proximal (arc I) and distal (arc II) cortical margins of the proximal carpal row and proximal carpal surfaces (arc III) of the hamate and capitate are shown in a posteroanterior view of the wrist

force distribution across the wrist and is related to some ulnar pathologies. Several studies have reported an association of negative ulnar variance with Kienböck's disease [3-5]. Bonzar et al. confirmed such an association after correcting for the influence of age on the measurement of ulnar variance [3]. However, D'Hoore and colleagues reported that negative ulnar variance does not seem to be an important factor in the etiology of Kienböck's disease [6]. Nakamura et al. found no significant difference in ulnar variance between Japanese subjects with normal wrists and those affected by Kienböck's disease [7]. The relationship between Kienböck's disease and negative ulnar variance remains controversial. On the other hand, positive ulnar variance plays a significant role in TFCC tears and ulnar impaction syndrome [2, 8-11]. Chronic impaction between the ulnar head and the TFCC and ulnar carpus results in the ulnar impaction syndrome, where degenerative tearing of the TFCC and chondromalacia of the lunate, triquetrum, and distal ulnar head are seen [2].

The crescentic lunate bone articulates with the scaphoid laterally, the triquetrum medially, the radius proximally, and the capitate distally. Smooth contiguous arcs form at the proximal and distal edges of the scapholunate and lunotriquetral joints. Interruption of the arcs should suggest fracture. The lunate may have a narrow facet at the distal medial aspect, which articulates with the hamate (type 2 lunate) (Fig. 2). Malik et al reported a type 2 lunate in one-half of 186 wrists [12]. A previous cadaveric study found the incidence of type 2 lunates to be as high as $73 \%$ [13]. Other studies found the prevalence of type 2 lunates to be $50-57 \%[14,15]$. The study by Malik and co-workers demonstrated that the average extra facet size of type 2 was $4.6 \mathrm{~mm}$ (range
$1.2-12.0 \mathrm{~mm}$ ) and apposition (articulation) of the extra lunate facet with the hamate averaged $77.4 \%$ (range $0-100 \%$ ) [12]. On magnetic resonance (MR) imaging, type 2 lunates usually have an innocuous appearance. A large percentage of patients with type 2 lunates also have chondromalacia, which often is occult on MR images [12]. The scapholunate and lunotriquetral intrinsic wrist ligaments anchor the lunate. Additionally, two major extrinsic wrist ligaments, the radiolunotriquetral and short radiolunate ligaments, attach to the volar aspect of the lunate.

The triquetrum articulates with the hamate at its distal aspect, with the lunate at the lateral aspect, and with the pisiform at its volar aspect. The triquetrum also articulates with the triangular fibrocartilage at its proximal aspect. The triquetrohamate joint is a helical joint, which permits the triquetrum to rotate around the hamate and also has a limited amount of translation distally, in the fashion of a screw. Most triquetrohamate joints lie on a continuous plane with the capitolunate joint and are described as type 1 ; in $20 \%$ of patients the triquetrohamate joint is separated from the capitolunate joint by a hamatolunate facet, described as a type 2 lunate [16].

The pisiform articulates with the triquetrum at its posterior surface and provides attachments to the flexor carpi ulnaris, abductor digiti quinti, and the transverse carpal ligament. The pisotriquetrum is a synovial joint that lies anterior to the remainder of the carpus and is susceptible to diseases that affect synovial joints. The synovium of the pisotriquetral joint does not communicate with the radiocarpal compartment at arthrography $12-25 \%$ of the time [17, 18]. The pisiform and pisotriquetral joint are best evaluated via the 'ball catcher' or 'allstate' views.

The distal radioulnar joint (DRUJ) is a pivot joint which permits rotation of the distal radius about the ulna during forearm pronation and supination. From pronation to supination, the ulnar head rotates approximately $150^{\circ}$ counterclockwise in relation to the radius. The DRUJ is stabilized by the volar radioulnar ligament, the dorsal radioulnar ligament, and the TFCC. The volar and dorsal radioulnar ligaments are narrow bands of fibers which extend, respectively, from the anterior and posterior margins of the ulnar notch of the radius to the ulna. There is an isolated synovial lining about the DRUJ which extends proximally between the radius and ulna [19]. On true lateral radiographs, the distal radius and ulna should be overlapping. If the radius and ulna are not superimposed while the carpal bones are seen to overlap, the suggestion is of a DRUJ injury.

\section{Ligaments}

The lunotriquetral ligament (LTL) is U-shaped on sagittal section and has three separate zones: dorsal, proximal, and 
Fig. 2 Type 2 lunate. a Type 1 lunate articulates only with the capitate distally (white arrow), while (b) type 2 lunate has articulation with the capitate and hamate (open arrow)
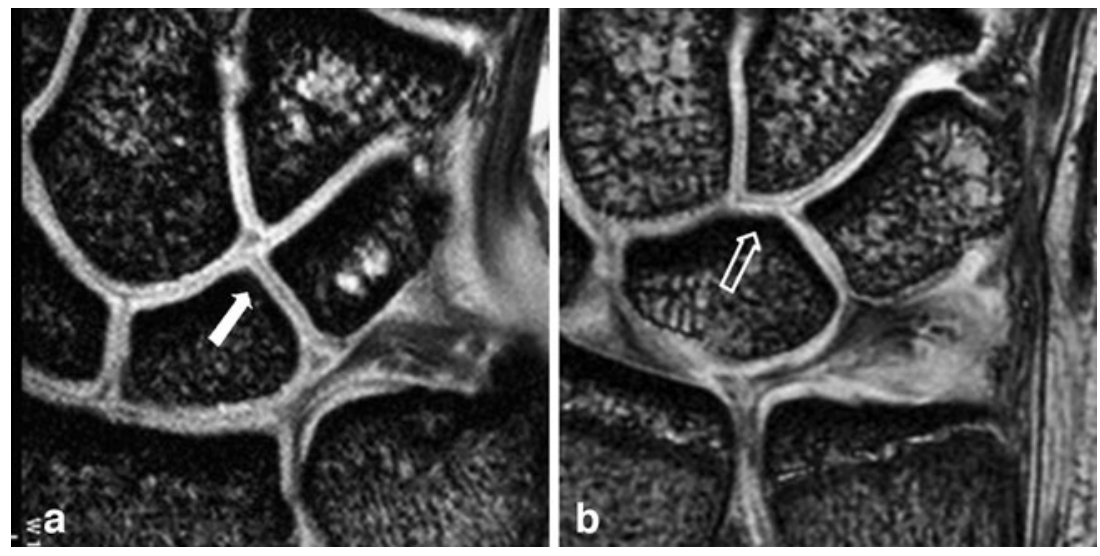

volar. The dorsal and volar zones are ligamentous. The former is highly important functionally as a restraint to rotation, while the latter is the strongest and thickest of all three zones and transmits the extension movement of the triquetrum [20,21]. The proximal zone is fibrocartilaginous and thin [20]. The radiographic appearance of wrists with LTL tears is often normal. Lunotriquetral dissociation will result in disruption of the smooth arcs that are formed by the proximal and distal joint surfaces of the proximal carpal row (Gilula's arcs I and II) and the proximal joint surfaces of the distal carpal row (Gilula's arc III). Arthrography can be helpful in demonstrating leakage or pooling of the contrast medium at the lunotriquetral interspace. However, age-related perforations of the LTL proximal zone, other communications between the radiocarpal and midcarpal joints, and asymptomatic LTL tears on arthrography of the normal wrist have been reported.

The dorsal and volar portions of the LTL are biomechanically more important than the proximal portion of the LTL. Therefore, images in the axial plane may be more helpful for assessing LTL injuries, although many previous MR studies have discussed LTL injuries in the coronal plane. The potential cause for false positive MR findings of the LTL tear is lack of familiarity with the normal MR appearance at the ligament-bone interface [22]. Smith and Snearly reported that the proximal zone of the LTL showed a variety of shapes and signal intensities [22]. Yoshioka et al. also recently reported the shape and signal intensity of the proximal zone of the LTL on a high-resolution two-dimensional (2D) gradient recalled echo (GRE) T2*-weighted sequence and emphasized the importance of familiarity with the normal MR appearance of the LTL proximal zone on high-resolution MR images to avoid inaccurate diagnosis of LTL injury (Fig. 3) [23]. The LTL proximal zone has a triangular shape in $>85 \%$ of subjects on GRE images. The linear intermediate or high signal intensity traversing the distal surface of the proximal LTL or traversing both distal and proximal surfaces of the proximal LTL is seen in approximately two-thirds of subjects [23]. The amorphous shape of the proximal LTL may represent diffuse degenerative changes [22]. The morphologic and signal intensity characteristics of the LTL interface with the lunate and triquetral bones are variable [22].

Triangular fibrocartilage complex

The TFCC is a fibrocartilage-ligament complex which stabilizes the DRUJ, transmits axial load between the carpus and the ulna, and stabilizes the ulnar aspect of the carpus. The TFCC is composed of the disc proper (articular disc), meniscus homologue, ulnolunate ligament, ulnotriquetral ligament, proximal ligamentous component, volar and dorsal radioulnar ligaments, and the ulnar collateral ligament. Because these structures are located in small areas, highresolution imaging is, we believe, essential to diagnose TFCC injuries.

In coronal MR images, the disc proper, triangular ligament, meniscus homologue, and ulnotriquetral ligament are clearly appreciated (Fig. 4), and, in the axial plane, volar and dorsal radioulnar ligaments are well recognized. The normal disc proper shows an asymmetrical bowtie-shaped low signal intensity. The signal intensity of the ulnar attachment of the TFCC was higher than that of the disc proper on proton density-weighted images and T2*-weighted images [24]. Most of the TFCC has two attachments to the ulna, inserting into the ulnar styloid tip (distal lamina) and the fovea (proximal lamina). The tissue between two triangular ligaments is called ligamentum subcruentum.

Three main arterial branches supply the TFCC: (1) the ulnar artery, (2) the palmar branch of the anterior interosseous artery, and (3) the dorsal branch of the anterior interosseous artery. The dorsal branch of the anterior interosseous artery runs on the dorsal side, while the palmar branch and the ulnar artery run on the palmar side. These three arteries supply blood to the periphery of the TFCC in a radial fashion (Fig. 5). Histologic sections demonstrate that the vessels penetrate the peripheral $15-20 \%$ of the disc, while the central portion and 

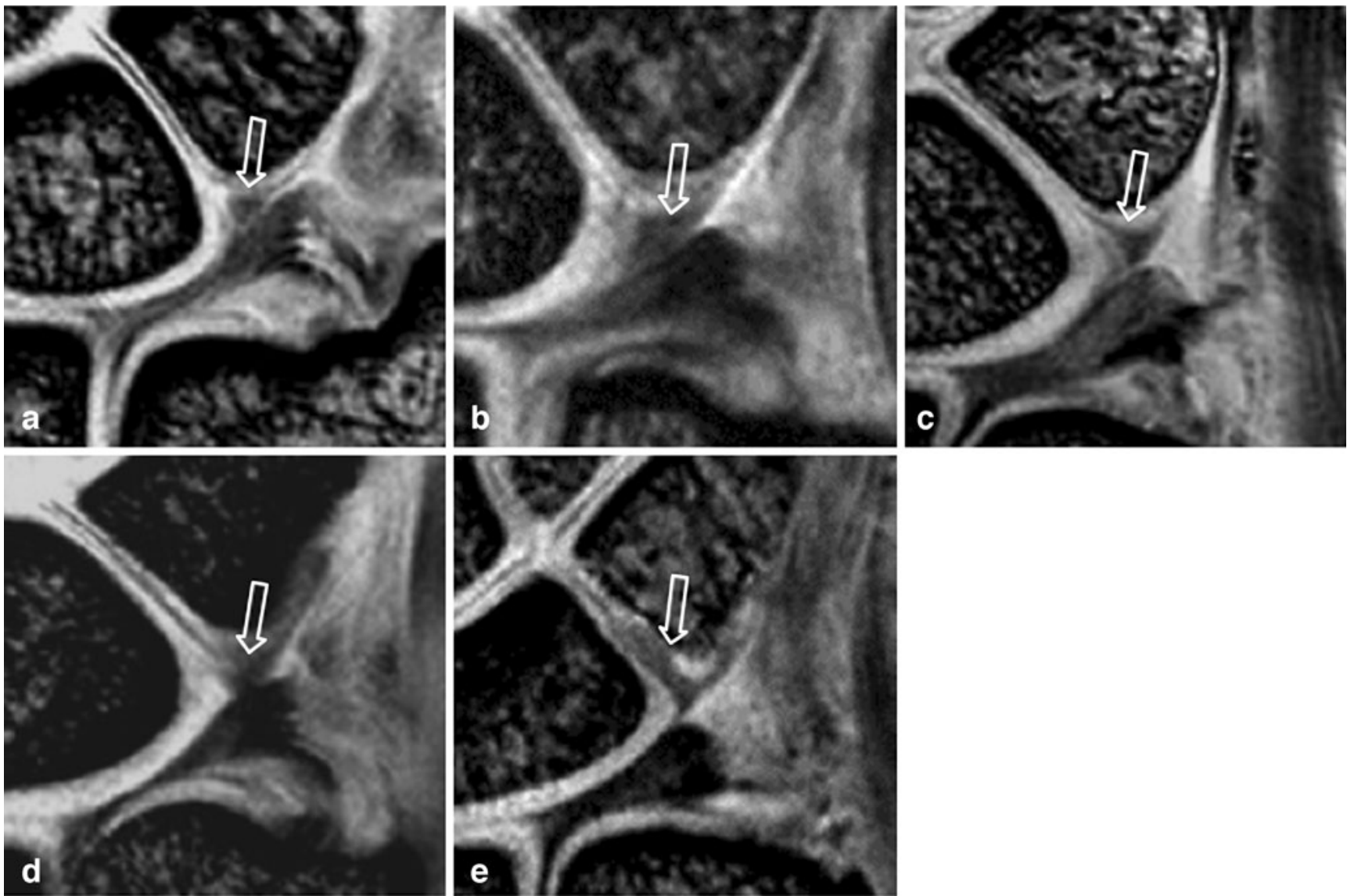

Fig. 3 The proximal zone of the LTL shows a broad spectrum of normal variations in shape on a $2 \mathrm{D}$ GRE T2*-weighted sequence [time to repeat/ time to echo (TR/TE)and flip angle 270-608/13-18 $\mathrm{ms}$ and $40^{\circ}$ ] (arrows). a Type 1, regular triangle; b type 2, broad-based triangle; c

type 3, narrow-based triangle; d type 4, asymmetrical triangle; e type 5, bar shape). (The figure has been reproduced from Yoshioka et al. Highresolution MR imaging of the proximal zone of the lunotriquetral ligament with a microscopy coil. Skeletal Radiol 2006;35:288-294)

radial attachment are avascular and consist mainly of chondrocytes in a fibrocartilaginous matrix. Therefore, tears of the TFCC in its vascular zone can heal if repaired, while those in the central avascular zone cannot [25]. The

vascularity and cellularity are more prominent in infants than in adults.

TFCC morphology is significantly associated with ulnar variance. The TFCC with zero ulnar variance is slightly

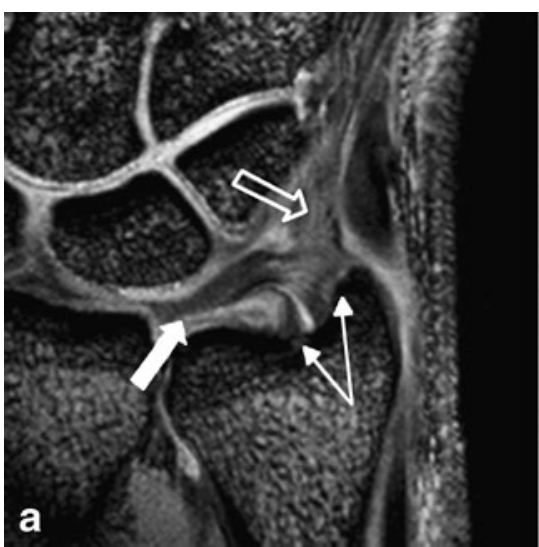

Fig. 4 High-resolution magnetic resonance imaging (MRI) of the TFCC. a Coronal 2D GRE T2*-weighted sequence [time to repeat/ time to echo (TR/TE) and flip angle $385 / 11 \mathrm{~ms}$ and $40^{\circ}$, a $1.5 \mathrm{~mm}$ slice thickness, a $50 \mathrm{~mm}$ field of view (FOV), and an imaging matrix

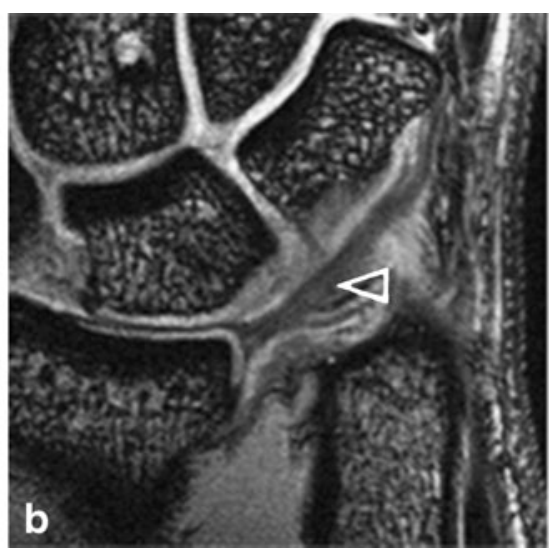

of 224 pixels $\times 512$ pixels] demonstrates the disc proper (thick white arrow), two laminae of triangular ligaments (thin arrows), and meniscus homologue (open arrow). b The ulnotriquetral ligament (arrowhead) is appreciated in a more volar slice 


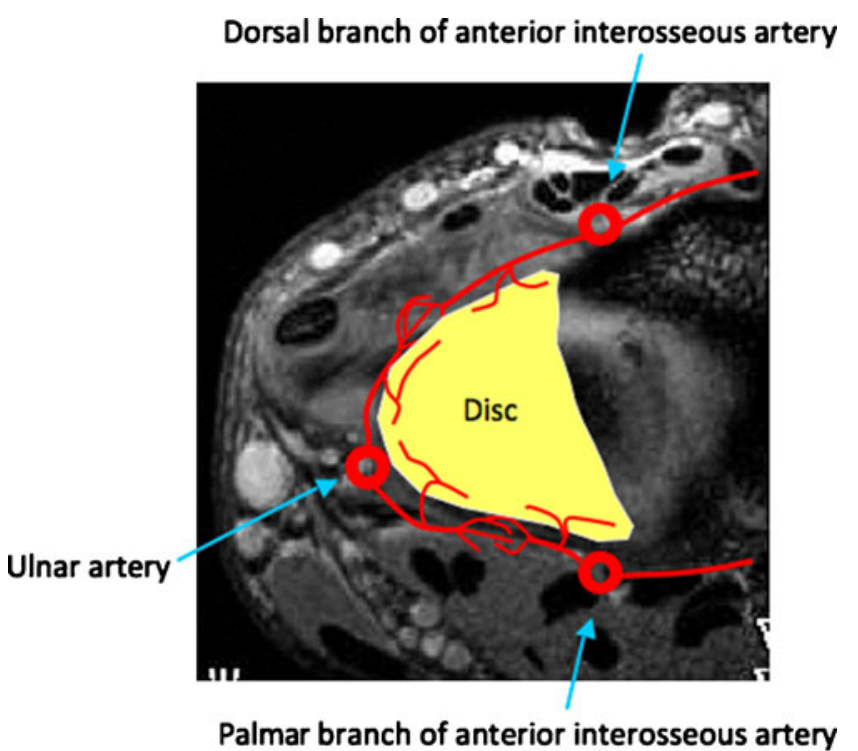

Fig. 5 Three main arterial branches supply the TFCC: (1) the ulnar artery, (2) the palmar branch of the anterior interosseous artery, and (3) the dorsal branch of the anterior interosseous artery. These arteries supply blood to the periphery of the TFCC in a radial fashion

tilted to a horizontal line at the level of the lunate fossa cartilage surface of the radius. The TFCC with minus variance runs more horizontally and is thicker and shorter than the TFCCs with zero variance and plus variance (Fig. 6). The TFCC with plus variance is thin, stretched distally, and arc-shaped between the ulnar head and proximal carpus. Therefore, there is an inverse relationship between ulnar variance and thickness of the TFCC. The ulnar-plus variance leads to ulnocarpal impaction and yields greater biomechanical forces, particularly rotational forces, in the disc compartment of the joint [26]. TFCC thickness appears to be related to the size of the available space between the ulnar head and the carpal bones, and a thin TFCC would be more vulnerable to biomechanical forces.
Yoshioka et al. reported that high-resolution MR images clearly demonstrated TFCC and cartilage of the wrist and ulnar variance [27]. The mean ulnar variance on magnetic resonance imaging (MRI) was $+0.26 \mathrm{~mm}$ (range $-4.59 \mathrm{~mm}$ to $+3.71 \mathrm{~mm}$ ). The mean TFCC angle and TFCC thickness were $23.9^{\circ}$ (range $-4.6^{\circ}$ to $+54.1^{\circ}$ ) and $1.11 \mathrm{~mm}$ (range $0.4 \mathrm{~mm}$ to $3.22 \mathrm{~mm}$ ), respectively. Ulnar variance and TFCC angle were positively correlated $(\mathrm{r}=0.84)$, and ulnar variance and TFCC thickness were negatively correlated $(\mathrm{r}=-0.71)$ (Fig. 7). However, ulnar variance and lunate or ulnar head cartilage thickness were not significantly correlated [27].

\section{Nerve and artery}

The ulnar nerve and artery pass through the wrist via a fibro-osseous tunnel medial to the carpal tunnel. This structure is known as the distal ulnar tunnel or Guyon's canal and extends from the pisiform to the hook of the hamate, approximately $4 \mathrm{~cm}$ distance [28]. The proximal Guyon's canal is triangular, with boundaries formed by the palmar carpal ligament at the volar aspect, pisiform at the ulnar aspect, and flexor retinaculum of the carpal tunnel at the radial and deep aspect (Fig. 8). The middle portion of Guyon's canal, between the pisiform and hook of hamate, is bordered at the volar aspect by the palmar carpal ligament, at the deep aspect by the pisohamate ligament, at the ulnar aspect by the abductor digiti minimi muscle, and at the radial aspect by the flexor retinaculum of the carpal tunnel. Within the middle portion of the canal, the ulnar nerve and artery bifurcate into deep and superficial branches. The distal Guyon's canal is defined by the palmaris brevis muscle at the volar and radial edge, the abductor digiti minimi muscle at the ulnar edge, and the flexor retinaculum and the hook of the hamate form the deep margin. At the distal Guyon's canal, the flexor digiti minimi brevis muscle

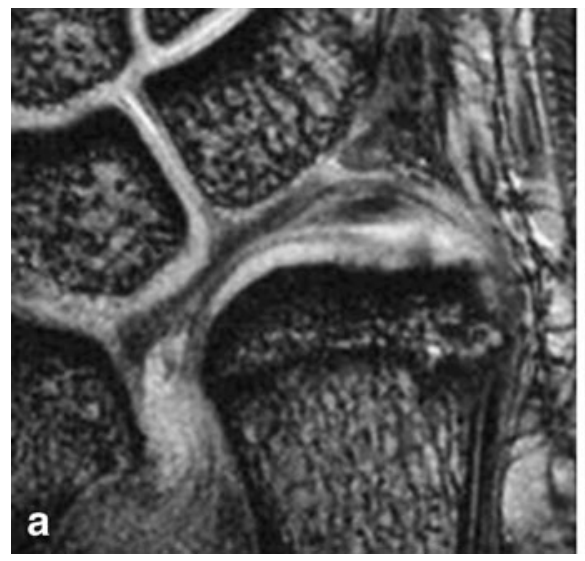

Fig. 6 Ulnar variance. a Positive, b neutral, c negative ulnar variance can be clearly demonstrated with high-resolution MRI. (Figure reproduced from Yoshioka et al. Study of ulnar variance with high-
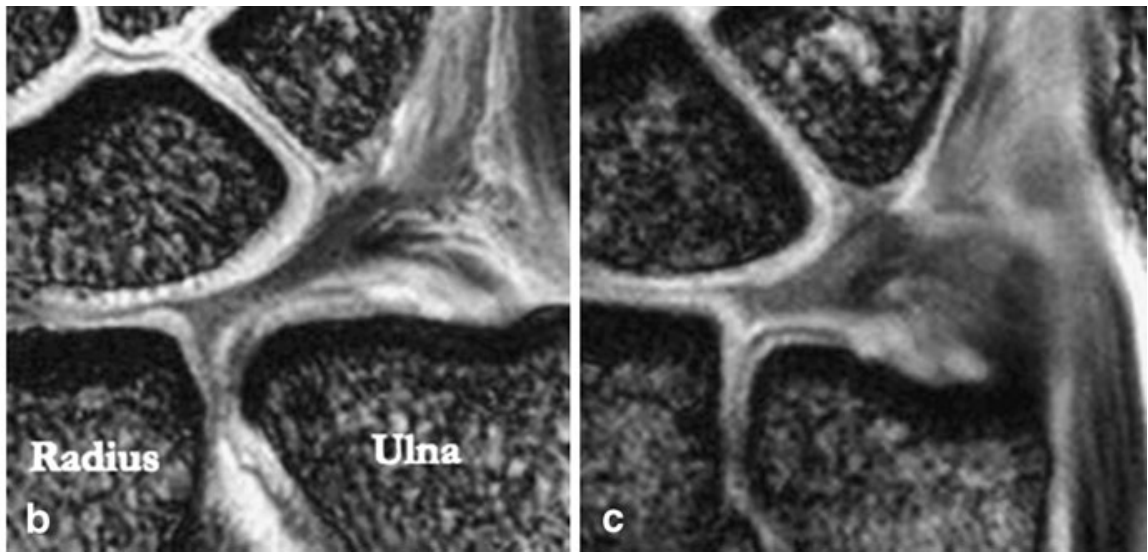

resolution MRI: correlation with triangular fibrocartilage complex and cartilage of ulnar side of wrist. J Magn Reson Imaging 2007;26:714 719) 
Fig. 7 Scattergrams of a ulnar variance versus TFCC angle and b ulnar variance versus TFCC thickness. Ulnar variance and TFCC angle were positively correlated, and ulnar variance and TFCC thickness were negatively correlated. (Figure reproduced from Yoshioka et al. Study of ulnar variance with high-resolution MRI: correlation with triangular fibrocartilage complex and cartilage of ulnar side of wrist. J Magn Reson Imaging 2007;26:714-719) a

Ulnar variance vs TFCC thickness

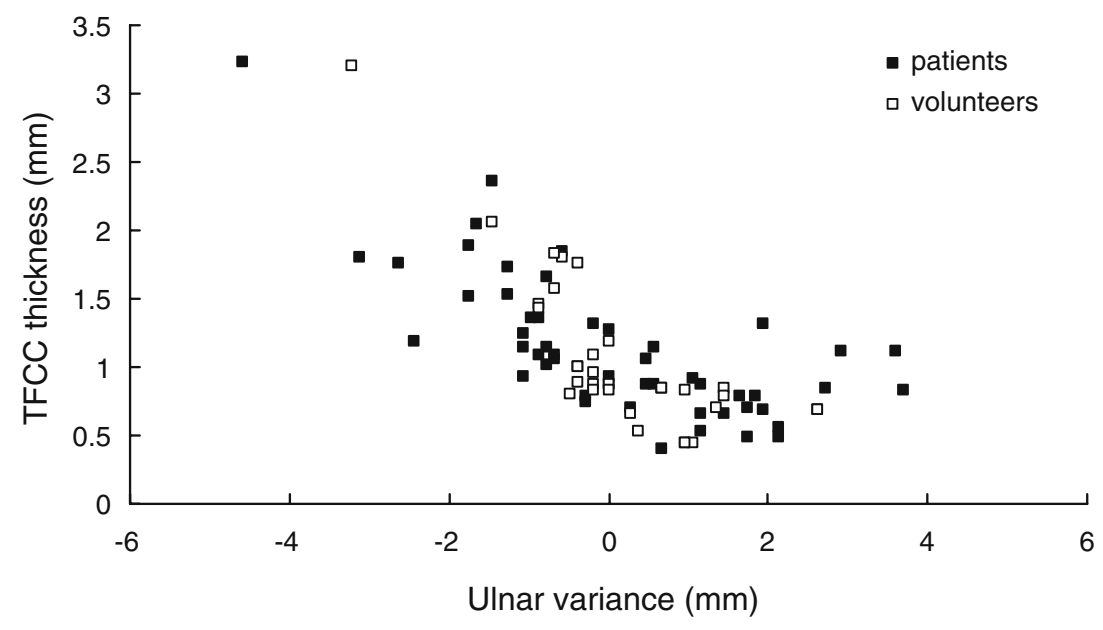

b

Ulnar variance vs TFCC angle
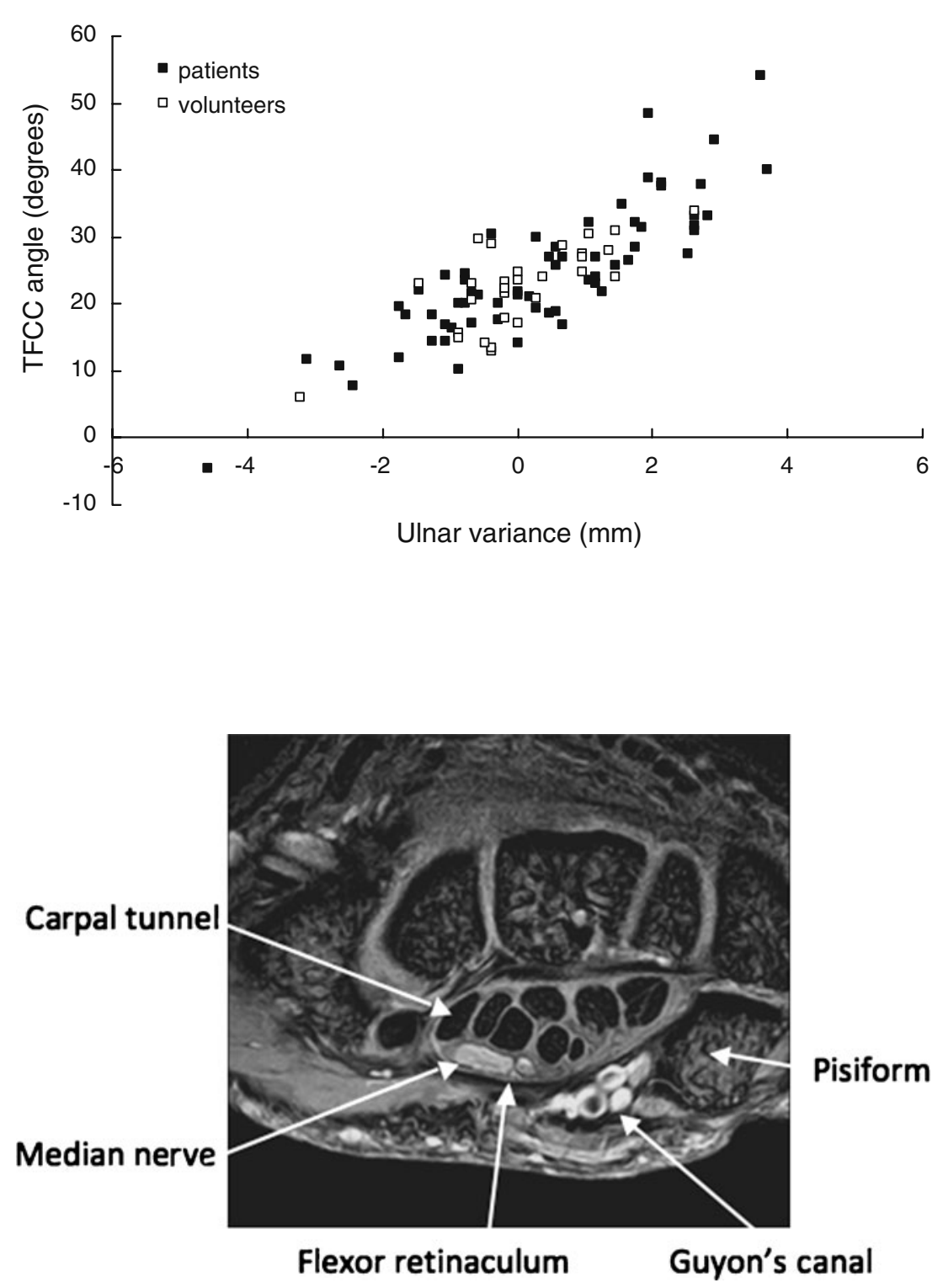

Flexor retinaculum
Guyon's canal
Fig. 8 Guyon's canal. The canal is located radial to the pisiform and volar to the flexor retinaculum of the carpal tunnel 
attaches to the hook of the hamate or a fibrous arch of the muscle resulting in a canal, containing the deep ulnar nerve and artery and a superficial compartment. Immediately distal to Guyon's canal, the superficial ulnar artery passes volar and adjacent to the hook of the hamate. This position adjacent to bone, without overlying protective structures, predisposes the superficial ulnar artery to traumatic injury in this location.

\section{Tendons}

The extensor carpi ulnaris (ECU) tendon lies within the sixth extensor compartment at the wrist, along a groove at the dorsolateral aspect of the ulna, and is covered by an annular ligament. The ECU tendon inserts distally at the midportion of the fifth metacarpal. In normal wrists, The ECU tendon may be partially displaced in the dorsal direction with supination and wrist extension and in the volar direction with pronation and wrist flexion [29, 30] (Fig. 9). The flexor carpi ulnaris (FCU) tendon lies at the ulnar aspect of the ulnar nerve and artery within the distal forearm proximal to Guyon's canal. The FCU tendon inserts on the pisiform and partially blends with the palmar carpal ligament forming the volar boundary of Guyon's canal.

\section{Clinical examination}

\section{History}

The clinical examination of a patient who presents for evaluation of ulnar-sided wrist pain begins with a thorough history. Hand dominance should be noted. A detailed past medical history that includes previous injury to the affected wrist, as well as other musculoskeletal involvement in the same extremity or in other regions of the body, may give important information that helps the clinician to arrive at an accurate diagnosis. Previous surgical procedures should be noted. The patient's social history may significantly influence the etiology, treatment, and prognosis. The social history should focus on profession and avocations. It is important to inquire about repetitive movements that the patient may make in work or hobbies, as certain movements may be the source of the pathology or may contribute to ongoing symptoms [31]. Worker's compensation status may affect outcome and expected postoperative rehabilitation [32].

The history of present illness must encompass several elements. The clinician should inquire about a history of traumatic injury. The time course of the symptoms should focus on sudden versus gradual onset as well as an acute versus chronic history. A detailed account of the mechanism of injury will give important diagnostic clues into the underlying pathology. For example, DRUJ and TFCC injuries often occur when the wrist is subjected to hyperpronation and axial loading forces [33]. The quality and intensity of pain may also suggest specific etiologies. Any associated numbness or paresthesias must be noted. Exacerbating and alleviating factors as well as radiation of pain is part of a complete history of present illness. The presence or absence of swelling, erythema, and tenderness may also assist in focusing the clinician's diagnostic evaluation. The onset of deformity must be inquired. For example, a wrist deformity that spontaneously resolved may suggest DRUJ instability [33]. Previous treatments and the response to these treatments, including occupational therapy and injections, provide valuable diagnostic information.

\section{Inspection}

The physical examination of a patient with ulnar-sided wrist pain begins with inspection. Symmetry of the wrist compared with that of the contralateral extremity and symmetry of the ulnar-sided digits compared with that of the radial-sided digits should be noted. Erythema, cyanosis, and edema may be present and should be noted. A focal area of edema around the wrist may suggest a ganglion. Previous surgical or traumatic scars are important to note. Atrophy can be assessed by comparison with the contralateral extremity, and its presence is suggestive of nerve pathology. Atrophy of the hypothenar
Fig. 9 The ECU tendon (white arrows) with (a) supination and (b) pronation. In a healthy subject without wrist pain the ECU tendon is partially displaced in the dorsal direction with supination and reduces with pronation
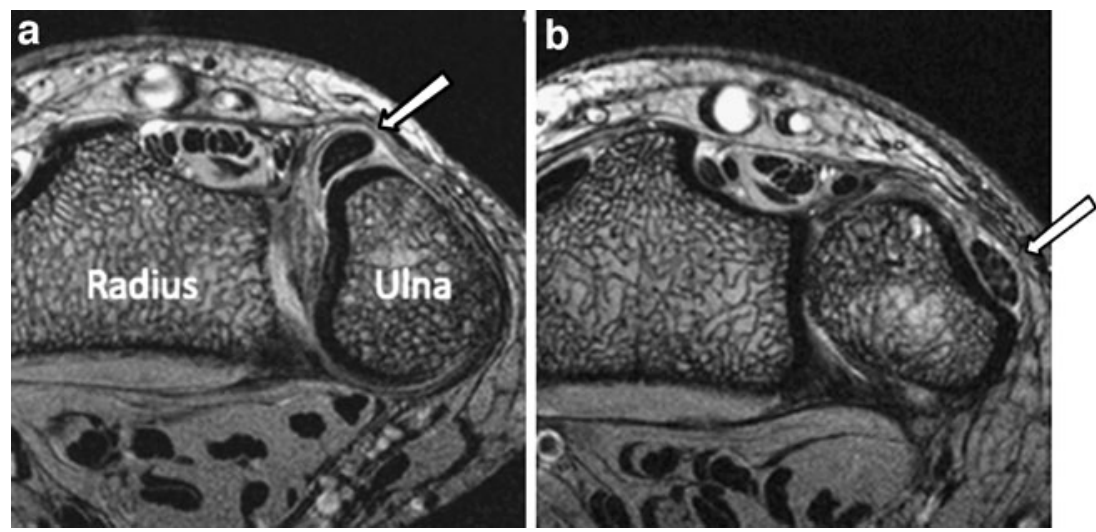
eminence may result from ulnar nerve compression in Guyon's canal or more proximally and can be expected to cause sensory disturbances in the ulnar nerve distribution [34].

\section{Range of motion}

Determination of range of motion is the next step in the physical examination. Both active and passive ranges of motion should be determined and compared with those of the contralateral side. Wrist flexion and extension from neutral are tested first. The clinician may expect normal wrist flexion of $80^{\circ}$ from neutral and wrist extension of $70^{\circ}$ from neutral [34]. Forearm supination and pronation should then be assessed with the patient's elbow flexed to $90^{\circ}$. Normal pronation of $90^{\circ}$ and supination of $90^{\circ}$ can be expected [34]. Range of motion of the digits should also be examined. The clinician should expect approximately $90^{\circ}$ of flexion and $30^{\circ}$ of extension at the metacarpophalangeal joints. The proximal interphalangeal joints are able to be ranged from $0^{\circ}$ to $100^{\circ}$, and the distal interphalangeal joints have a normal range of motion from $20^{\circ}$ of extension to $90^{\circ}$ of flexion [34]. Range of motion of the elbow should be examined, with normal flexion to $135^{\circ}$ and normal extension to $0^{\circ}$. Shoulder and neck motion should be measured if deemed necessary based on the clinician's judgment.

\section{Vascular examination}

Vascular examination should begin with palpation of the radial and ulnar artery pulses. The ulnar artery is contained within Guyon's canal, which is a fibro-osseus canal bordered by the pisiform ulnarly and the hook of hamate radially [35]. The clinician may palpate Guyon's canal volarly to examine the ulnar nerve and artery. Particular attention should be paid to the presence of a ganglion, softtissue mass, or aneurysm. Inability to palpate an ulnar pulse may indicate ulnar artery thrombosis that can result from trauma to the hypothenar eminence [36]. An Allen test should be performed to examine the patency of the ulnar and radial arteries. For this test, the patient opens and closes the fist vigorously several times in order to exsanguinate blood from the hand. With the patient's hand tightly closed in a fist, the examiner occludes both arteries simultaneously by compressing the arteries against the underlying bones. The patient is instructed to open his or her hand. The examiner then releases pressure from either the ulnar or radial artery and observes blood flow into the pale hand. Capillary refill of the palm and digits should be brisk after pressure has been released. This maneuver is then repeated to assess patency of the other artery [37]. Capillary refill of the digits should also be examined. Normal capillary refill takes fewer than $5 \mathrm{~s}$ [37].

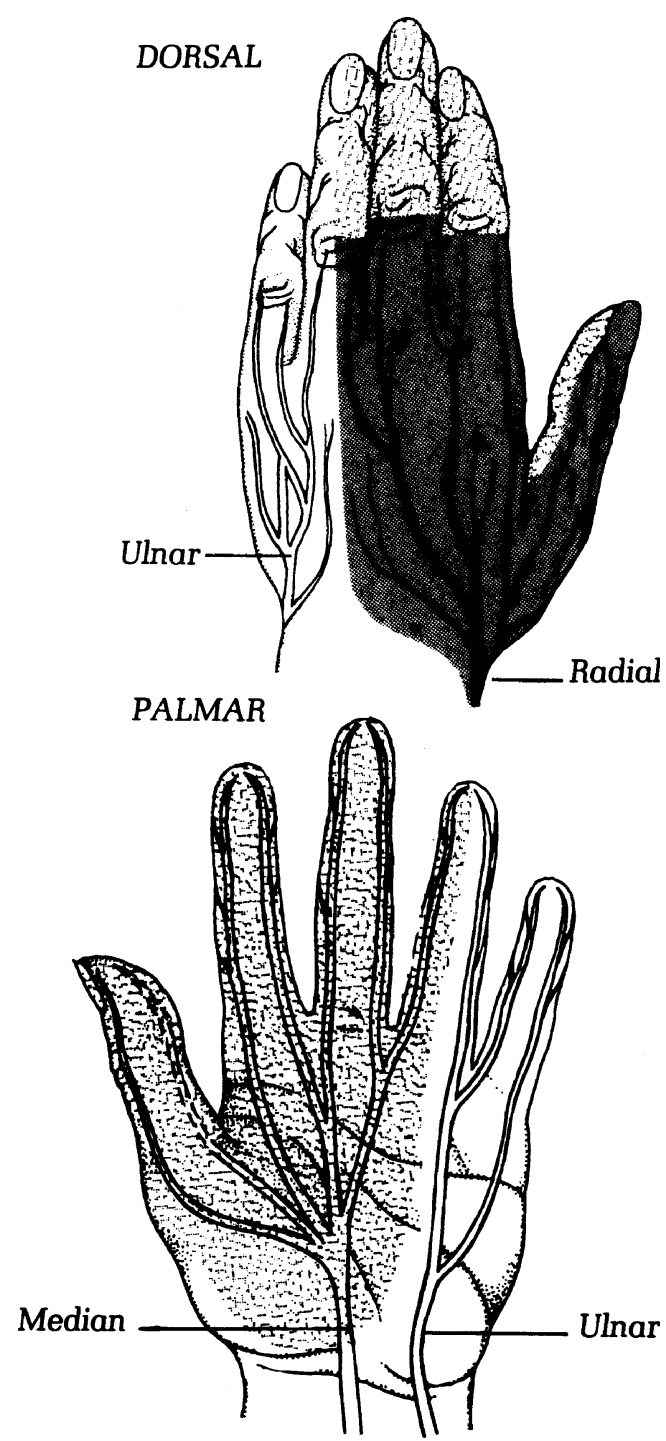

Fig. 10 Sensory distributions of the median, ulnar, and radial nerves. (Reprinted with permission of the American Society for Surgery of the Hand. The hand: examination and diagnosis, 3rd edition. New York: Churchill Livingstone, 1990)

Neurologic examination

Neurologic examination of the patient with ulnar-sided wrist pain should include thorough interrogation of sensory and motor function. Sensory testing includes two-point discrimination in the distributions of the median, radial, and ulnar nerves (Fig. 10). The median nerve supplies sensation to the skin over the thenar eminence and the palmar aspects of the thumb, index, and long fingers as well as the distal dorsal aspects of these digits [34]. Sensation of this nerve is often tested clinically by examination of the sensation of the palmar skin of the index finger. The radial nerve innervates the dorsal aspects of the thumb, index finger, and ring finger as far as the distal interphalangeal (DIP) joints, 
the dorsum of the hand on the radial side of the long finger metacarpal, including the dorsal web space between the thumb and index fingers [37]. Sensory testing of the first dorsal web space between the thumb and index finger is an effective way of examining radial nerve integrity. The ulnar nerve supplies sensation to the dorsal and radial surfaces of the ulnar aspects of the wrist, hand, ring finger, and small finger. Clinicians often test sensation in the ulnar nerve distribution by sensory examination of the volar distal aspect of the small finger [34].

Motor examination begins with the testing of motor function of muscles innervated by the median, radial, and ulnar nerves. The median nerve and its branch, the anterior interosseus nerve, are examined by the testing of the abductor pollicis brevis muscle, the flexor digitorum superficialis and profundus muscles, and the flexor pollicis longus. The radial nerve and its branch, the posterior interosseus nerve, are examined by the testing of wrist and digital extension. Digital abduction demonstrates function of the dorsal interosseus muscles and examines the ulnar nerve. Wrist extension and flexion, as well as wrist ulnar and radial deviation, must be carefully examined in the patient with ulnar-sided wrist pain. Wrist extension is tested by stabilizing the patient's forearm with one hand and placing pressure on the dorsum of the patient's wrist with the other hand, while the patient is instructed to extend the wrist [34]. The muscles that contribute to wrist extension include the extensor carpi radialis longus (ECRL) and brevis (ECRB) and the ECU. The ECRL and ECRB run through the second dorsal compartment of the wrist (Fig. 11). When the patient extends and radially deviates the wrist, these two tendons can be palpated on the dorsoradial aspect of the wrist [37]. To evaluate the ECU, the clinician must ask the patient to extend and ulnarly deviate the wrist. With this motion the ECU tendon can be easily palpated on the dorsal and ulnar aspect of the wrist [37]. When examining wrist flexion the clinician should ensure that the patient is making a fist to prevent the finger flexors from acting as a wrist flexor [34]. The flexor carpi radialis (FCR), palmaris longus, and flexor carpi ulnaris (FCU) are the muscles that are active in wrist flexion and can be palpated during the testing of wrist flexion [37].

Palpation

Upon completion of the neurologic examination, the clinician should utilize palpation to examine osseus structures, articulations, and soft tissue structures for point tenderness, crepitus, and swelling. As described earlier, the osteology and articulations of the ulnar side of the wrist and
Fig. 11 Extensor compartments of the wrist. The ECRL and ECRB are contained within the second dorsal compartment. $\mathrm{MC}=$ metacarpal, APL $=$ abductor pollicis longus, $\mathrm{EPB}=$ extensor pollicis brevis, $\mathrm{EPL}=$ extensor pollicis longus, $\mathrm{EIP}=$ extensor indicis proprius, $\mathrm{EDC}=$ extensor digitorum communis, $\mathrm{EDM}=$ extensor digiti minimi. (Reprinted with permission from Trumble T, Principles of hand surgery and therapy. Philadelphia: Saunders, 2000)
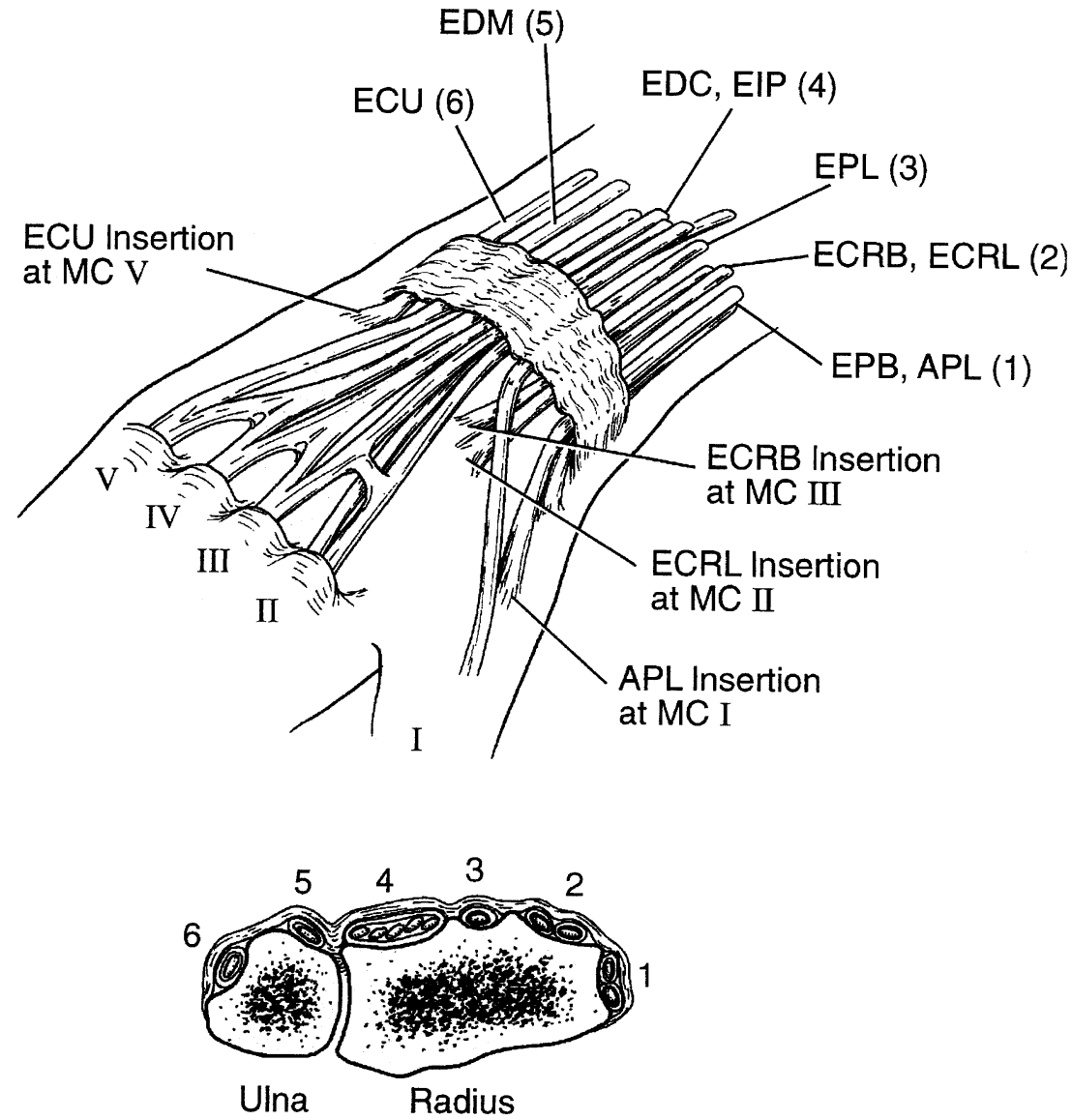
Fig. 12 Radiographs of DRUJ dislocation. a Anteroposterior (AP) view and $\mathbf{b}$ lateral view of a right wrist demonstrating dislocation of the DRUJ. This injury results from hyperpronation with damage to the dorsal radioulnar ligament of the TFCC and dorsal capsule of the DRUJ. A dorsal prominence over the wrist may be evident on physical examination

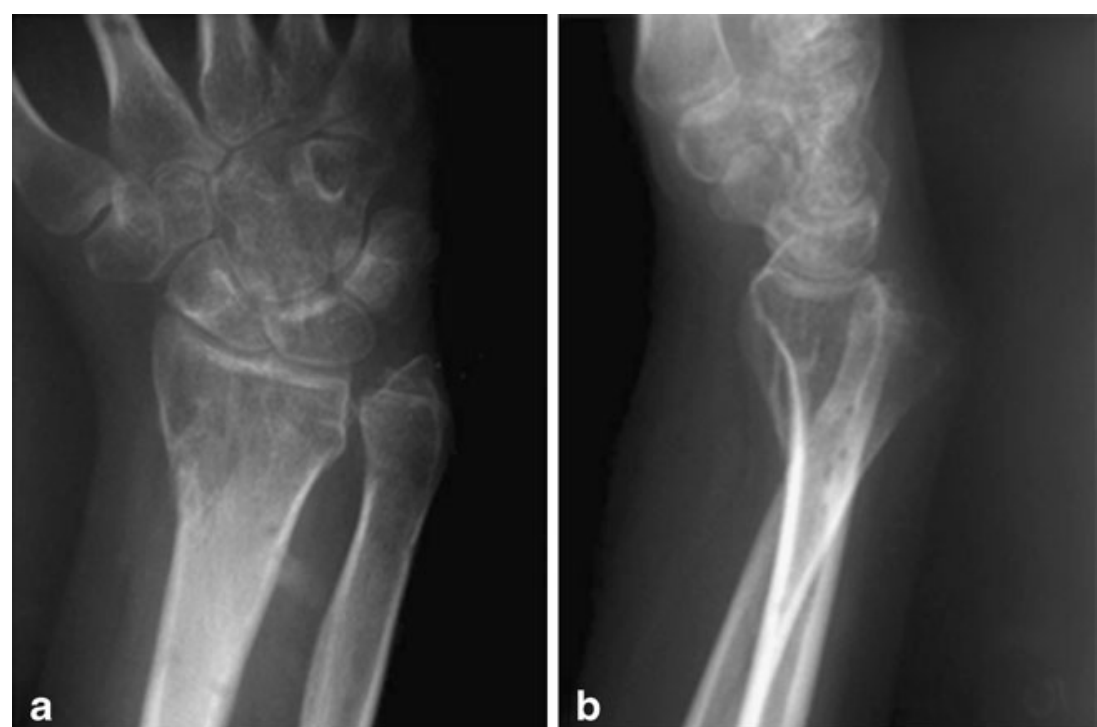

hand are complex. One method of examination includes progression from proximal to distal. First the distal ulna, including the ulnar styloid, is palpated. The DRUJ is a frequent site of ulnar-sided wrist pain pathology. Acute injuries to the DRUJ result in tenderness and loss of wrist rotation [38]. Dorsal dislocation of the DRUJ is the result of a hyperpronation injury with damage to the dorsal radioulnar ligament of the TFCC and dorsal capsule of the DRUJ [38] (Fig. 12). This injury may also present with a dorsal prominence over the wrist and with the forearm locked in pronation.

After examination of the ulna and DRUJ, the carpal bones should be examined. The lunate is just proximal to the capitate and is the most frequently dislocated bone in the wrist [34]. It is the second most frequently fractured bone in the wrist [34]. Flexion and extension of the patient's wrist allows the examiner to assess the motion of the capitolunate articulation
[34]. Lunate tenderness, especially in association with decreased range of motion of the wrist, may indicate Kienböck's disease [39] (Fig. 13). The triquetrum can be palpated distal to the ulnar styloid by radial deviation of the patient's wrist [37]. The lunotriquetral interval is palpable dorsally between the fourth and fifth extensor compartments one finger's breadth distal to the DRUJ with the wrist in $30^{\circ}$ of flexion [33]. Limited range of motion, point tenderness, laxity, and a snap over the lunotriquetral (LT) joint suggest a lunotriquetral ligament sprain or complete tear. Dorsopalmar manipulation of the triquetrum on the lunate demonstrates crepitus and laxity [40]. The pisiform is contained within the FCU tendon at the level of the palmar crease, and this carpal bone can be located by palpation distally along the FCU tendon [34, 41]. Owing to this location, the pisiform is subjected to high compressive and shear forces [42]. Direct compression and wrist flexion, a motion often performed in

Fig. 13 Radiographs showing Kienböck's disease. a) Anteroposterior (AP) view and $\mathbf{b}$ lateral view of the right wrist demonstrating lunate necrosis characteristic of Kienböck's disease. Physical examination often reveals tenderness in the area of the lunate as well as decreased range of motion of the wrist
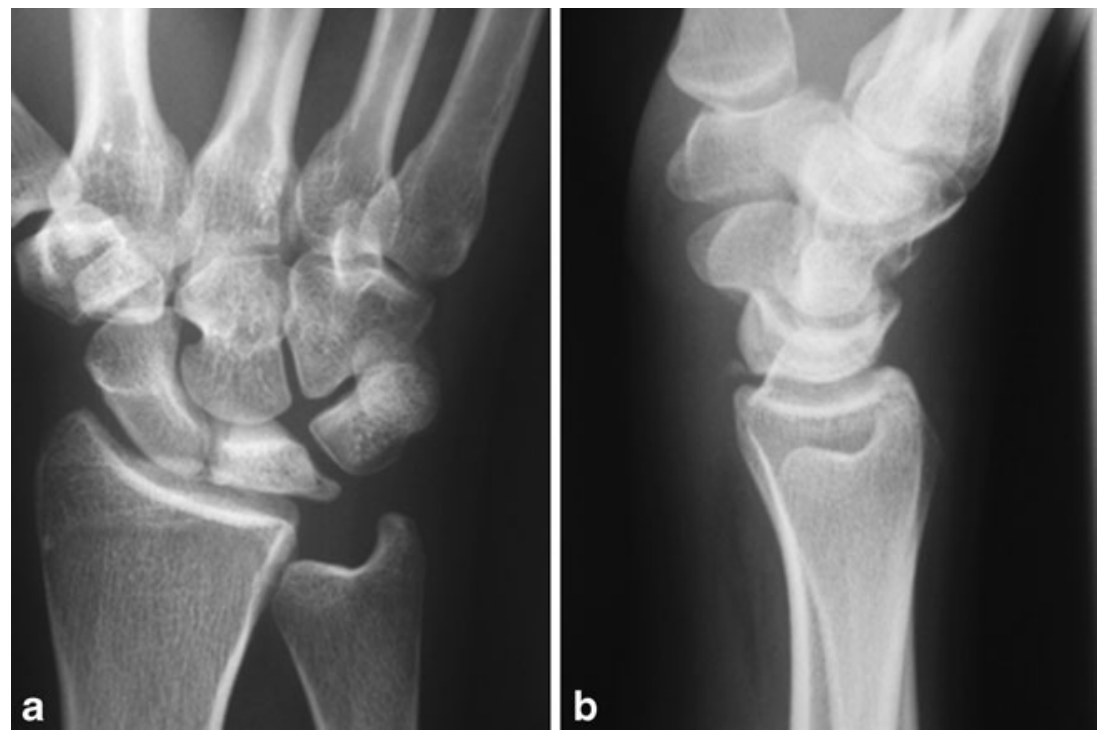
racquet sports, can aggravate pisotriquetral arthritis [36]. Lidocaine injection into the pisotriquetral joint can help to confirm the diagnosis of pisotriquetral arthritis [36].

Within the distal carpal row, the capitate is palpable immediately proximal to the base of the middle finger metacarpal. When the wrist is in the neutral position, a small depression is palpable in the region of the capitate, and flexion of the wrist causes this depression to roll distally [34]. Located ulnar to the capitate is the hamate. The body of the hamate can be palpated dorsally, while the hook of hamate can be examined with volar palpation. The hook of hamate is located by palpation from the pisiform towards the head of the second metacarpal [34]. This structure is located along a line drawn between these two structures approximately $2 \mathrm{~cm}$ from the pisiform. To complete palpation of the osseous structures, the clinician should examine the base of the ring finger and small finger metacarpals.

Following osseous examination, the soft tissues of the ulnar aspect of the wrist are examined. Dorsally, the tendon of the ECU is contained within the sixth dorsal compartment of the wrist, which is a fibro-osseous tunnel over the most distal aspect of the ulna [43]. Large forces are applied at the sixth dorsal compartment; thus, the ECU is at risk of rupture at this location. The ECU tendon should be examined for subluxation over the ulnar styloid process $[44,45]$. This may occur with pronation of the wrist after trauma that results in a tear of the carpal ligament of the sixth dorsal compartment [45-48]. An audible snap may have been heard or experienced by the patient [34]. Rheumatoid arthritis may also predispose an individual to ECU subluxation or rupture [34] (Fig. 14). On the volar and ulnar aspect of the wrist, the FCU should be examined. This tendon is more prominent with abduction and extension of the digits. The TFCC, which is located on the ulnar border of the wrist, just distal to the head of the ulna, can be located by palpation of the depression between the pisiform, FCU, and ulnar styloid [49, 50].

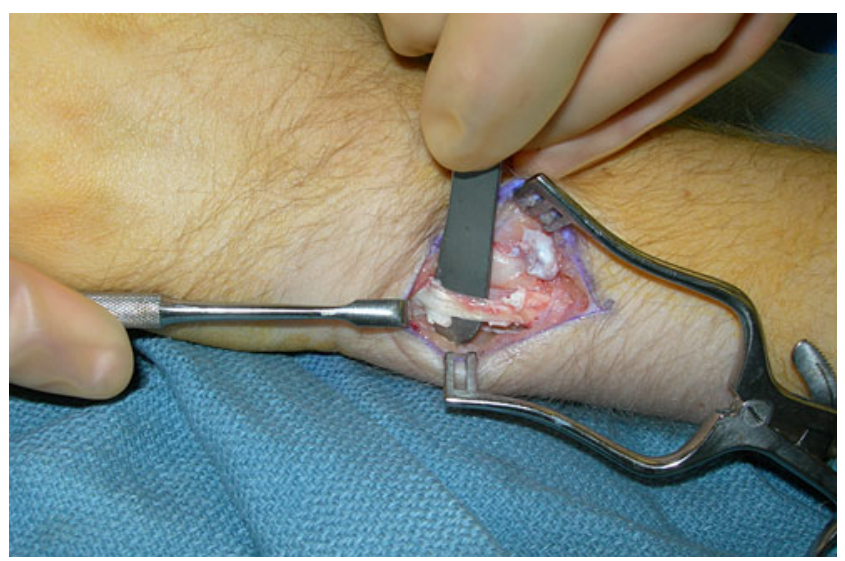

Fig. 14 ECU tenosynovitis with partial rupture. Rheumatoid arthritis may predispose an individual to ECU subluxation and rupture
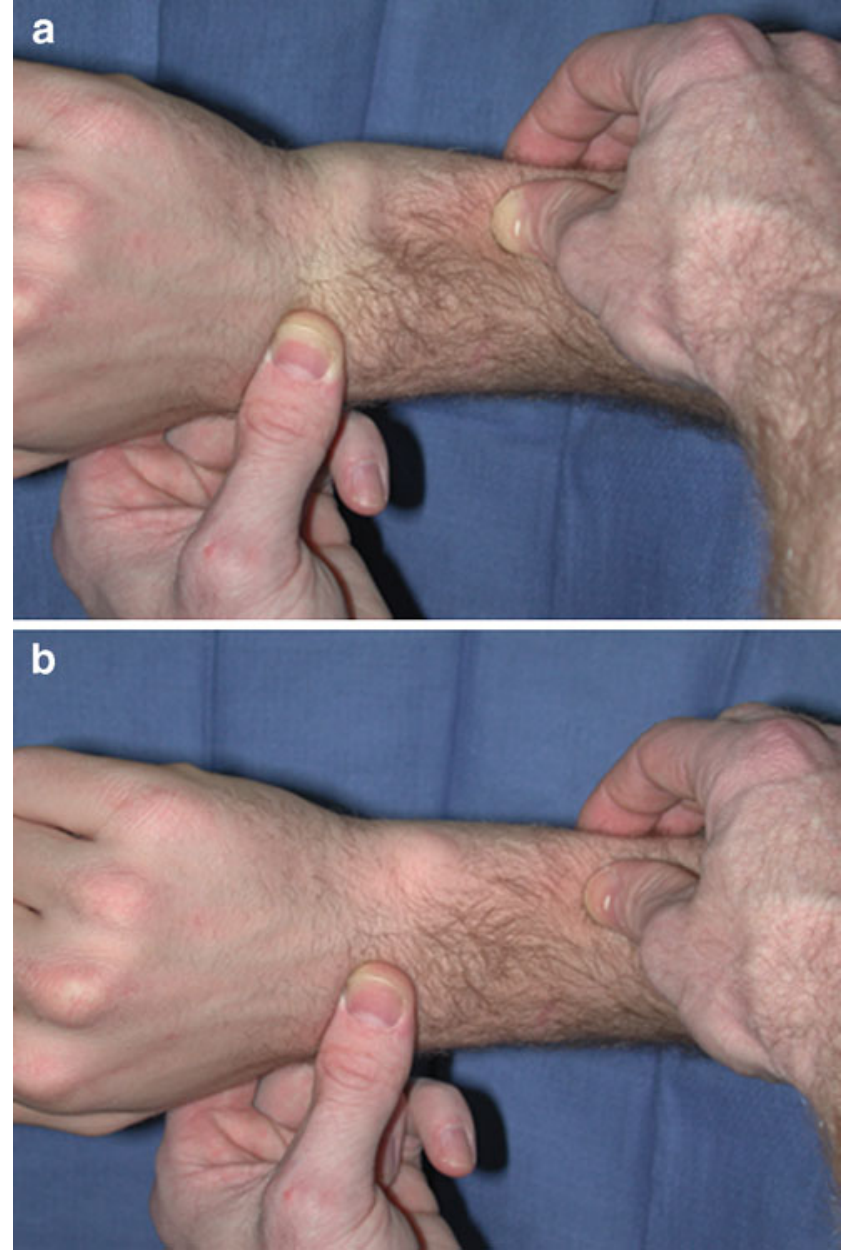

Fig. 15 Piano-key test for investigation of DRUJ stability. a This test is performed by the examiner's supporting the wrist in pronation and applying force to the ulnar head. b The piano-key test shows a positive result if the ulnar head returns to its normal anatomic position when the force is removed from the ulnar head

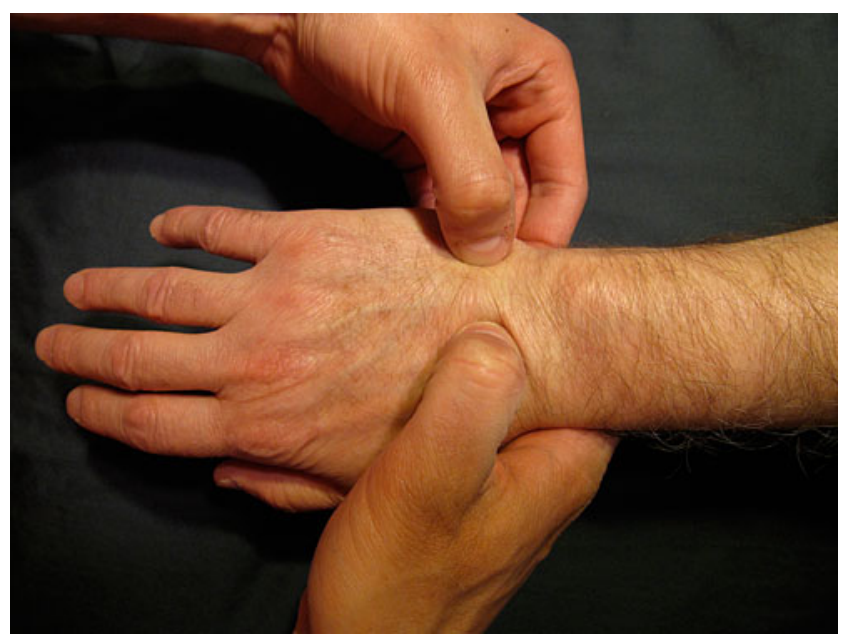

Fig. 16 Lunotriquetral ballottement test to examine the lunotriquetral joint. Force is applied to the lateral border of the triquetrum to compress the lunate against the triquetrum 


\section{Provocative maneuvers}

There are a number of specialized physical examination maneuvers that may aid the diagnosis of ulnar-sided wrist pain. The ulnocarpal stress test, as described by Nakamura et al., is performed by the examiner's placing the wrist in maximum ulnar deviation while applying an axial load and passively pronating and supinating the wrist [51, 52]. Pain elicited by this maneuver may be indicative of ulnar-sided wrist pain; for example, pain secondary to ulnocarpal impaction syndrome for which the test was originally described [51]. Although a sensitive test for ulnar-sided wrist pain, the ulnocarpal stress test is not specific for ulnocarpal impaction syndrome. Lunotriquetral ligament injury, triangular fibrocartilage tears, and ulnocarpal arthritis will produce positive findings in the ulnocarpal stress test; thus, this test may best be used as a screening tool for intra-articular pathology $[52,53]$.

The ulnar fovea sign is elicited by palpation of the 'soft spot' bordered by the ulnar styloid process dorsally, the flexor carpi ulnaris tendon volarly, the volar surface of the ulnar head proximally, and the pisiform distally [54]. Significant tenderness that reproduces the patient's complaint in terms of character and location is considered a positive finding of the ulnar fovea sign. This sign is a sensitive test for foveal disruptions and ulnotriquetral ligament injuries [54].

Stability of the DRUJ can be investigated with the use of the piano-key test (Fig. 15). In this test the wrist is supported in pronation and force is applied to the ulnar head. The piano-key test result is positive if the ulnar head returns to its normal anatomic position when the force is removed from the ulnar head, just as a piano key springs up when force is released from the key [37]. The ulnar compression test is also useful in examining the DRUJ. This maneuver is performed with compression of the ulnar head against the sigmoid notch. Pain caused by the ulnar compression test suggests inflammation, arthritis, or instability of the DRUJ [55].

The lunotriquetral ballottement test is one of several provocative maneuvers that assess the lunotriquetral joint $[40,56]$ (Fig. 16). Force is applied to the lateral border of the triquetrum to compress the lunate against the triquetrum. The ulnar snuffbox test is another valuable maneuver used to examine the triquetrum and lunotriquetral joint. In this maneuver radial pressure is applied to the sulcus between the ECU tendon and the flexor carpi ulnaris tendon; reproduction of the pain is considered to be a positive finding and may suggest triquetral chondromalacia or lunotriquetral injury [56].

Midcarpal instability can be investigated with the midcarpal shift test. The patient's forearm is stabilized in a pronated position in $15^{\circ}$ of ulnar deviation. With the examiner's thumb placed at the distal aspect of the patient's capitate, volar pressure is applied to the capitate while an axial load is applied to the metacarpals. A painful clunk that reproduces the patient's symptoms is considered to be a positive finding for the midcarpal shift test [57].

The pisotriquetral grind test is useful in examining the pisotriquetral joint. To perform this maneuver the examiner moves the pisiform in an ulnar and radial direction, using the thumb and index finger along with compression of the pisiform. Pain elicited by this maneuver is considered to be a positive result of the test and suggests pisotriquetral arthritis $[58,59]$.

Open Access This article is distributed under the terms of the Creative Commons Attribution Noncommercial License which permits any noncommercial use, distribution, and reproduction in any medium, provided the original author(s) and source are credited.

\section{References}

1. Metz VM, Wunderbaldinger P, Gilula LA. Update on imaging techniques of the wrist and hand. Clin Plast Surg. 1996;23:369-84.

2. Cerezal L, del Pinal F, Abascal F, Garcia-Valtuille R, Pereda T, Canga A. Imaging findings in ulnar-sided wrist impaction syndromes. Radiographics. 2002;22:105-21.

3. Bonzar M, Firrell JC, Hainer M, Mah ET, McCabe SJ. Kienbock disease and negative ulnar variance. J Bone Joint Surg Am. 1998;80:1154-7.

4. Gelberman RH, Salamon PB, Jurist JM, Posch JL. Ulnar variance in Kienbock's disease. J Bone Joint Surg Am. 1975;57:674-6.

5. Mirabello SC, Rosenthal DI, Smith RJ. Correlation of clinical and radiographic findings in Kienbock's disease. J Hand Surg [Am]. 1987;12:1049-54.

6. D'Hoore K, De Smet L, Verellen K, Vral J, Fabry G. Negative ulnar variance is not a risk factor for Kienbock's disease. J Hand Surg [Am]. 1994;19:229-31.

7. Nakamura R, Tanaka Y, Imaeda T, Miura T. The influence of age and sex on ulnar variance. J Hand Surg [Br]. 1991;16:84-8.

8. Imaeda T, Nakamura R, Shionoya K, Makino N. Ulnar impaction syndrome: MR imaging findings. Radiology. 1996;201:495-500.

9. Mellado JM, Calmet J, Domenech S, Sauri A. Clinically significant skeletal variations of the shoulder and the wrist: role of MR imaging. Eur Radiol. 2003;13:1735-43.

10. Mikic ZD. Detailed anatomy of the articular disc of the distal radioulnar joint. Clin Orthop Relat Res. 1989(245):123-132.

11. Palmer AK. Triangular fibrocartilage complex lesions: a classification. J Hand Surg [Am]. 1989;14:594-606.

12. Malik AM, Schweitzer ME, Culp RW, Osterman LA, Manton G. MR imaging of the type II lunate bone: frequency, extent, and associated findings. AJR Am J Roentgenol. 1999;173:335-8.

13. Viegas SF, Patterson RM, Hokanson JA, Davis J. Wrist anatomy: incidence, distribution, and correlation of anatomic variations, tears, and arthrosis. J Hand Surg [Am]. 1993;18:463-75.

14. Pfirrmann CW, Theumann NH, Chung CB, Trudell DJ, Resnick D. The hamatolunate facet: characterization and association with cartilage lesions-magnetic resonance arthrography and anatomic correlation in cadaveric wrists. Skeletal Radiol. 2002;31:451-6.

15. Sagerman SD, Hauck RM, Palmer AK. Lunate morphology: can it be predicted with routine X-ray films? J Hand Surg [Am]. 1995;20:38-41. 
16. Burgess RC. Anatomic variations of the midcarpal joint. J Hand Surg [Am]. 1990;15:129-31.

17. Pessis E, Drape JL, Bach F, Feydy A, Guerini H, Chevrot A. Direct arthrography of the pisotriquetral joint. AJR Am J Roentgenol. 2006;186:800-4.

18. Theumann NH, Pfirrmann CW, Chung CB, Antonio GE, Trudell DJ, Resnick D. Pisotriquetral joint: assessment with MR imaging and MR arthrography. Radiology. 2002;222:763-70.

19. Shigematsu KOS, Takaoka T, Suzuki J, Okuda M. Distal radioulnar joint arthrography for detection of deep portion tears of the triangular fibrocartilage complex. J Jpn Soc Surg Hand. 2001;17:558-61.

20. Johnstone DJ, Thorogood S, Smith WH, Scott TD. A comparison of magnetic resonance imaging and arthroscopy in the investigation of chronic wrist pain. J Hand Surg [Br]. 1997;22:714-8.

21. Zlatkin MB, Chao PC, Osterman AL, Schnall MD, Dalinka MK, Kressel HY. Chronic wrist pain: evaluation with high-resolution MR imaging. Radiology. 1989;173:723-9.

22. Smith DK, Snearly WN. Lunotriquetral interosseous ligament of the wrist: MR appearances in asymptomatic volunteers and arthrographically normal wrists. Radiology. 1994;191:199-202.

23. Yoshioka H, Tanaka T, Ueno T, Shindo M, Carrino JA, Lang P, et al. High-resolution MR imaging of the proximal zone of the lunotriquetral ligament with a microscopy coil. Skeletal Radiol. 2006;35:288-94.

24. Tanaka T, Ogino S, Yoshioka H. Ligamentous injuries of the wrist. Semin Musculoskelet Radiol. 2008;12:359-77.

25. Thiru RG, Ferlic DC, Clayton ML, McClure DC. Arterial anatomy of the triangular fibrocartilage of the wrist and its surgical significance. J Hand Surg [Am]. 1986;11:258-63.

26. Sugimoto H, Shinozaki T, Ohsawa T. Triangular fibrocartilage in asymptomatic subjects: investigation of abnormal MR signal intensity. Radiology. 1994;191:193-7.

27. Yoshioka H, Tanaka T, Ueno T, Carrino JA, Winalski CS, Aliabadi $P$, et al. Study of ulnar variance with high-resolution MRI: correlation with triangular fibrocartilage complex and cartilage of ulnar side of wrist. J Magn Reson Imaging. 2007;26:714-9.

28. Zeiss J, Jakab E, Khimji T, Imbriglia J. The ulnar tunnel at the wrist (Guyon's canal): normal MR anatomy and variants. AJR Am J Roentgenol. 1992;158:1081-5.

29. Pfirrmann CW, Theumann NH, Chung CB, Botte MJ, Trudell DJ, Resnick D. What happens to the triangular fibrocartilage complex during pronation and supination of the forearm? Analysis of its morphology and diagnostic assessment with MR arthrography. Skeletal Radiol. 2001;30:677-85.

30. Pratt RK, Hoy GA, Bass Franzcr C. Extensor carpi ulnaris subluxation or dislocation? Ultrasound measurement of tendon excursion and normal values. Hand Surg. 2004;9:137-43.

31. Armstrong TJ, Fine LJ, Goldstein SA, Lifshitz YR, Silverstein BA. Ergonomics considerations in hand and wrist tendinitis. J Hand Surg [Am]. 1987;12:830-7.

32. Iwasaki N, Ishikawa J, Kato H, Minami M, Minami A. Factors affecting results of ulnar shortening for ulnar impaction syndrome. Clin Orthop Relat Res. 2007;465:215-9.

33. Sachar K. Ulnar-sided wrist pain: evaluation and treatment of triangular fibrocartilage complex tears, ulnocarpal impaction syndrome, and lunotriquetral ligament tears. J Hand Surg [Am]. 2008;33:1669-79.

34. Hoppenfeld S. Physical examination of the spine and extremities. Norwalk, Connecticut: Appleton \& Lange; 1976.
35. Murata K, Tamai M, Gupta A. Anatomic study of arborization patterns of the ulnar artery in Guyon's canal. J Hand Surg [Am]. 2006;31:258-63.

36. Buterbaugh GA, Brown TR, Horn PC. Ulnar-sided wrist pain in athletes. Clin Sports Med. 1998;17:567-83.

37. American Society for Surgery of the Hand. The hand: examination and diagnosis. 3rd ed. New York: Elsevier; 1990.

38. Buterbaugh GA, Palmer AK. Fractures and dislocations of the distal radioulnar joint. Hand Clin. 1988;4:361-75.

39. Schuind F, Eslami S, Ledoux P. Kienbock's disease. J Bone Joint Surg Br. 2008;90:133-9.

40. Reagan DS, Linscheid RL, Dobyns JH. Lunotriquetral sprains. J Hand Surg [Am]. 1984;9:502-14.

41. Jenkins SA. Osteoarthritis of the pisiform-triquetral joint; report of three cases. J Bone Joint Surg Br. 1951;33-B:532-4.

42. Beckers A, Koebke J. Mechanical strain at the pisotriquetral joint. Clin Anat. 1998;11:320-6.

43. Spinner M, Kaplan EB. Extensor carpi ulnaris. Its relationship to the stability of the distal radio-ulnar joint. Clin Orthop Relat Res. 1970;68:124-9.

44. Burkhart SS, Wood MB, Linscheid RL. Posttraumatic recurrent subluxation of the extensor carpi ulnaris tendon. J Hand Surg [Am]. 1982;7:1-3.

45. Rayan GM. Recurrent dislocation of the extensor carpi ulnaris in athletes. Am J Sports Med. 1983;11:183-4.

46. Chun S, Palmer AK. Chronic ulnar wrist pain secondary to partial rupture of the extensor carpi ulnaris tendon. J Hand Surg [Am]. 1987;12:1032-5.

47. Eckhardt WA, Palmer AK. Recurrent dislocation of extensor carpi ulnaris tendon. J Hand Surg [Am]. 1981;6:629-31.

48. Hajj AA, Wood MB. Stenosing tenosynovitis of the extensor carpi ulnaris. J Hand Surg [Am]. 1986;11:519-20.

49. Ahn AK, Chang D, Plate AM. Triangular fibrocartilage complex tears: a review. Bull NYU Hosp Jt Dis. 2006;64:114-8.

50. Palmer AK, Werner FW. The triangular fibrocartilage complex of the wrist — anatomy and function. J Hand Surg [Am]. 1981;6:153-62.

51. Friedman SL, Palmer AK. The ulnar impaction syndrome. Hand Clin. 1991;7:295-310.

52. Nakamura R, Horii E, Imaeda T, Nakao E, Kato H, Watanabe K. The ulnocarpal stress test in the diagnosis of ulnar-sided wrist pain. J Hand Surg [Br]. 1997;22:719-23.

53. Nakamura R. Diagnosis of ulnar wrist pain. Nagoya J Med Sci. 2001;64:81-91.

54. Tay SC, Tomita K, Berger RA. The "ulnar fovea sign" for defining ulnar wrist pain: an analysis of sensitivity and specificity. J Hand Surg [Am]. 2007;32:438-44.

55. Kleinman WB, Graham TJ. Distal ulnar injury and dysfunction. In: Peimer CA, editor. Surgery of the hand and upper extremity. New York: McGraw-Hill; 1996. pp. 667-709.

56. Ambrose L, Posner MA. Lunate-triquetral and midcarpal joint instability. Hand Clin. 1992;8:653-68.

57. Lichtman DM, Schneider JR, Swafford AR, Mack GR. Ulnar midcarpal instability - clinical and laboratory analysis. J Hand Surg [Am]. 1981;6:515-23.

58. Green DP. Pisotriquetral arthritis: a case report. J Hand Surg [Am]. 1979;4:465-7.

59. Carroll RE, Coyle MP Jr. Dysfunction of the pisotriquetral joint: treatment by excision of the pisiform. J Hand Surg [Am]. 1985;10:703-7. 